\title{
Well shaped ST segment and risk of cardiovascular mortality
}

\author{
Evert G Schouten, Jacqueline M Dekker, Jan Pool, Frans J Kok, Maarten L Simoons
}

\begin{abstract}
Objective-To investigate the prognostic value of frequently occurring slight variations in the ST segment for cardiovascular mortality in healthy subjects.

Design-Follow up study of mortality in relation to variations in ST segment level in a cohort over the 28 years from 1953 to 1981 . A case-cohort sampling design was applied to limit the number of electrocardiograms that had to be coded by hand.

Setting-General health examination carried out in 1953 of civil servants in Amsterdam and assessment of subsequent mortality.

Subjects-Apparently healthy civil servants aged 40 to 65 years: 1583 men and 1508 women.

Main outcome measures-Relative risk of variations in ST segment level for mortality from all causes, cardiovascular disease, and coronary heart disease.

Results - In men the multivariate relative risks of 15 year mortality from cardiovascular disease and coronary heart disease of slight ST elevation at $80 \mathrm{~ms}$ past the J point (compared with isoelectric ST segment) were 0.5 (95\% confidence interval 0.3 to $0.9)$ and $0.4(0.2$ to 0.8), respectively. As expected ST segment depression $(>0.25 \mathrm{~mm})$ was associated with increased risk: $1.9(1.1$ to 3.0$)$ and $2.2(1.2$ to 3.9 ), respectively. In women associations were weaker. The full 28 year period showed a similar pattern of somewhat weaker associations for men; among women, however, no predictive value was apparent.
\end{abstract}

Conclusion - These results are empirical evidence for the intuitive opinion among doctors that a curved, upward sloping ST segment, resulting in slight ST elevation at $80 \mathrm{~ms}$, indicates low risk compared with the isoelectric flat, stretched ST segment.

\section{Introduction}

In healthy subjects ST depressions in an electrocardiogram taken at rest and defined according to the Minnesota code ${ }^{1}$ are associated with increased risk of coronary events. ${ }^{2-9}$ However, no data have been reported about the risk associated with ST elevation in such subjects.

We studied small variations in the ST segment level, both depression and elevation, in relation to mortality from all causes, cardiovascular disease, and coronary heart disease during 28 years of follow up of 3091 apparently healthy subjects. Most of the small variations we analysed do not meet clinical or Minnesota criteria for cardiovascular disease and are commonly seen in healthy people. They may represent less favourable autonomic balance rather than coronary disease.

University, Rottendam,

Netherlands

Jan Pool, MD

Maarten L Simoons, MD

Correspondence to:

Dr Schouten. healthy civil servants of the city of Amsterdam and their spouses and were aged between 40 and 65 . The survey was carried out to study the feasibility and benefit of screening healthy middle aged people. About $54 \%$ of the 11700 eligible civil servants volunteered, with or without their spouses. The cohort of participants was a random sample stratified by age and sex from among the volunteers, comprising roughly equal numbers of subjects in five year age bands for both sexes. The information on survival or cause of death needed for our study was missing for 19 men and 12 women -in most instances because they were living abroad-leaving 1564 men and 1496 women. The cohort and aims of the original study have been reported in more detail elsewhere. ${ }^{10}$

As the coding of electrocardiograms was very time consuming, we did not study the whole cohort but used a case-cohort sampling design. ${ }^{11-13}$ With this approach cases (subjects who died during follow up) were compared with a random sample from the cohort instead of with a sample of non-case referents, as in a case-control design. We wanted the size of the random sample from the cohort to be twice the expected number of deaths from cardiovascular events at 28 years of follow up. We estimated that the proportion of deaths from cardiovascular disease was $40 \%$ of all deaths and took into account the differential in mortality between men and women. Our random sample comprised 717 men (46\% of the male cohort) and 494 women (33\% of the female cohort). Because the processing of the electrocardiograms had to start before the causes of death had been established, however, we included all other deaths in the remaining cohort. This resulted in a population of 1219 men and 848 women. Ten men and 14 women were excluded because information on the ST segment was missing. The final case-cohort study population was therefore 1209 men and 834 women, among whom there were, respectively, 887 and 508 deaths from all causes, 418 and 225 from cardiovascular disease, and 232 and 94 from coronary heart disease in the full 28 years of follow up.

\section{DATA COLLECTION}

The general health survey during 1953-4 consisted of a detailed medical history obtained by interview, a physical examination, and some laboratory examinations. ${ }^{14}$ At baseline during 1953-4 a 12 lead electrocardiogram was recorded on an Elema Mingograph one channel, ink jet recorder. Recently the electrocardiograms were classified according to the Minnesota code. ${ }^{1}$ Furthermore the level of the ST segment in lead I $80 \mathrm{~ms}$ past the J point relative to the baseline level was measured in movements of $0.25 \mathrm{~mm}(0.025 \mathrm{mV})$. Lead I was chosen because of its orientation to the left side of the heart and because this lead was recorded after light exercise as well.

In 1981 survival of $99.9 \%$ of the original population was established with the help of the civil servants' pension fund (ABP). ${ }^{10}$ In 1989 the cause of death up to 1981 was established for $98.0 \%$ of the men and $98 \cdot 1 \%$ of the women by using the registers of the Dutch Central Bureau of Statistics.

For the analysis mortality from all causes, cardio- 
vascular disease (ICD-9 codes 390-460), and coronary heart disease (ICD-9 codes 410-414) were selected. ${ }^{15}$

\section{DATA ANALYSIS}

Participants were classified according to four categories of ST segment level: ST elevated, ST isoelectric (reference category), ST depressed $0.25 \mathrm{~mm}$, and ST depressed $>0.25 \mathrm{~mm}$. Means and percentages of relevant baseline characteristics were calculated for categories of ST segment level in the random sample of the cohort (see table I).

A stratified analysis was performed to evaluate the effect of age, cardiovascular risk factors, blood pressure, serum cholesterol concentration, body mass index, and smoking as potential confounders. We used age strata with cut off points of 50 and 60 years and strata based on approximate tertiles for the other confounders.

Case-cohort relative risks (cumulative incidence

TABLE I-Baseline characteristics and mortality according to ST segment level in random sample of cohort. Values are means $(S D)$ unless stated otherwise

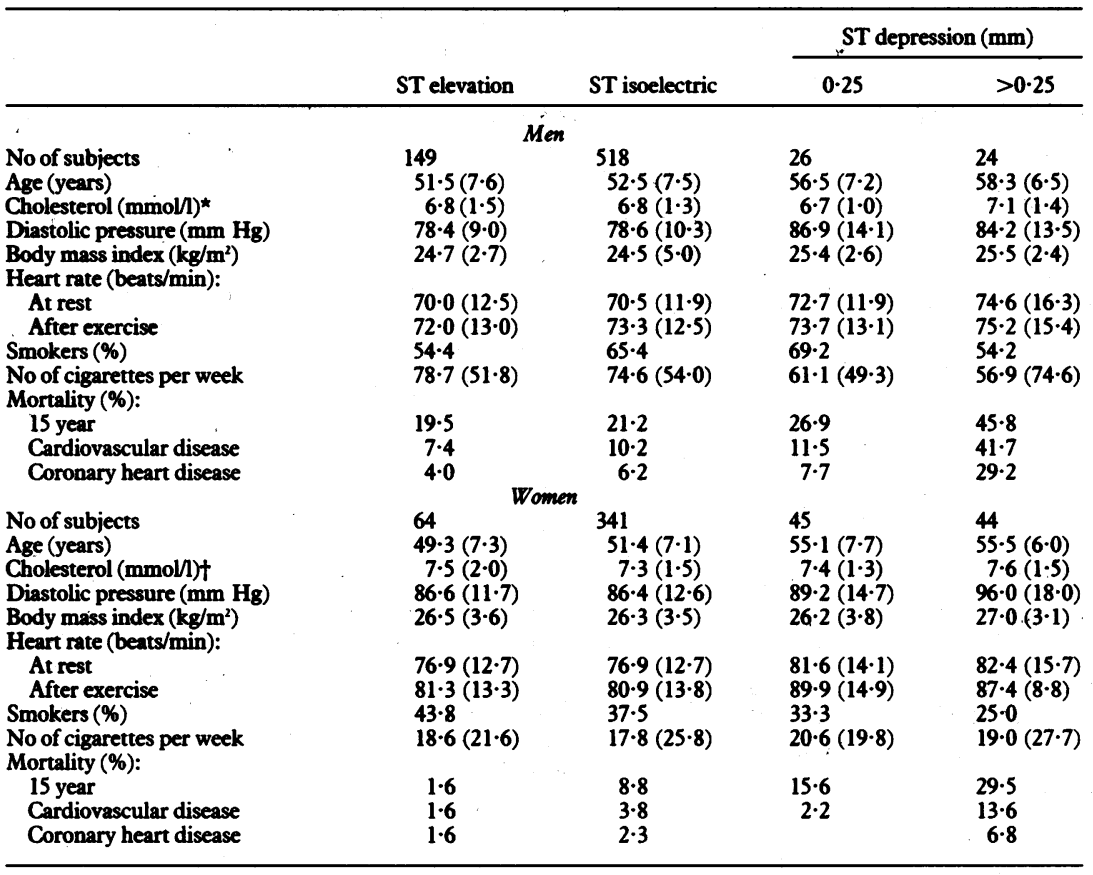

^Forty missing observations among men and nine among women.

TABLE II-Case-cohort relative risk of 15 year mortality from all causes, cardiovascular disease, and coronary heart disease by ST segment level, with ST isoelectric as reference category

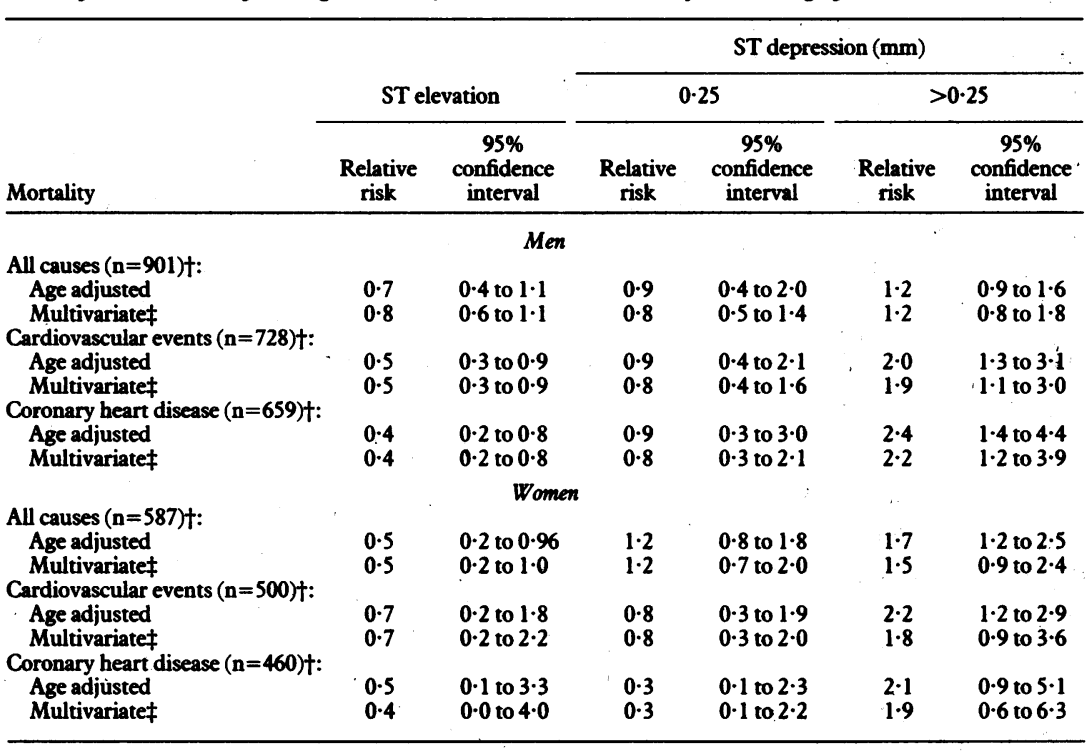

*Cases compared with random sample from cohort.

tSize of case-cohort population for given category of mortality.

†^djusted for age, total serum cholesterol concentration, diastolic blood pressure, smoking, and body mass index.

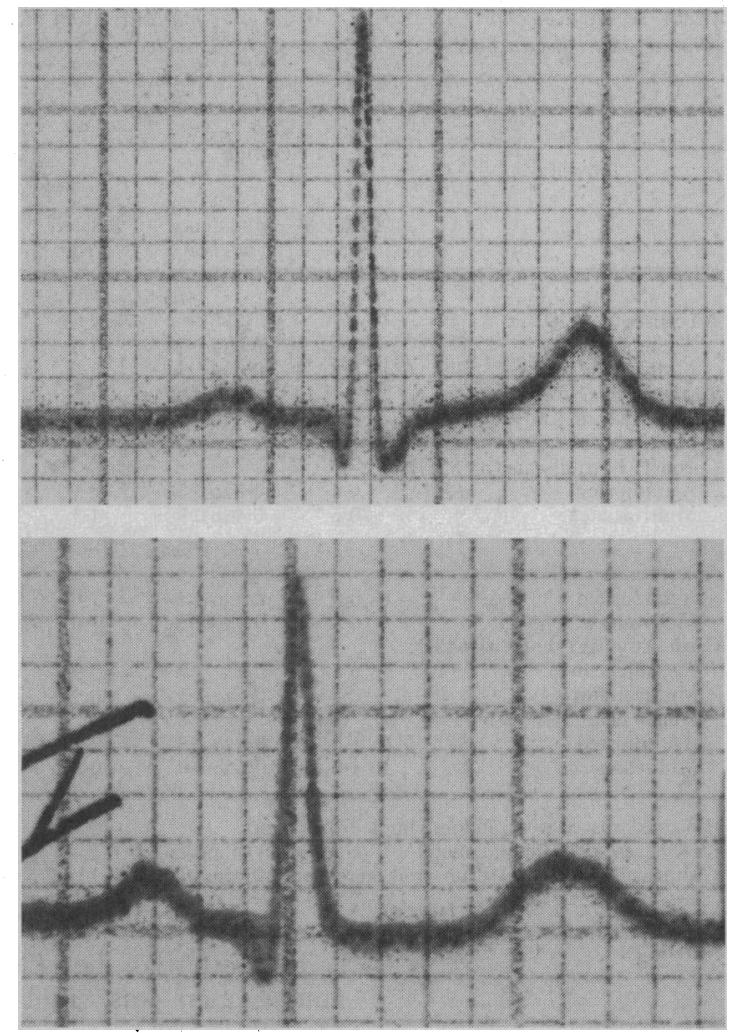

Example of well shaped ST segment with upward sloping curve (top) and of isoelectric, flat ST segment (bottom)

ratios) adjusted for age alone and their $95 \%$ confidence intervals were estimated. ${ }^{16}$ Multivariate estimation was carried out by means of logistic regression analysis, introducing categories of potential confounders as indicator variables. In the case-cohort analysis the odds ratio estimates true relative risk (cumulative incidence ratio) without needing the rare disease assumption. ${ }^{12}$ In all analyses the sample representing the cohort is identical while the number of deaths depends on the chosen cause of death category.

\section{Results}

Table I shows the baseline characteristics and mortality by categories of ST segment level in the random sample from the cohort. Age, diastolic blood pressure, and mortality showed the most important differences from values in the reference category (ST isoelectric), especially in the group with ST depression $>0.25 \mathrm{~mm}$.

The measurement of ST segment level in lead I yielded 214 men and 102 women with ST elevation in the case-cohort study population (210 and $102 \leqslant 1$ $\mathrm{mm})$. Fifty two men and 85 women had an ST depression of $0.25 \mathrm{~mm}$ and 42 men and 67 women a depression of $>0.25 \mathrm{~mm}$. The figure shows typical examples of a slightly elevated (well shaped) ST segment and of an isoelectric (flat) ST segment.

Multivariate relative risks of ST elevation compared with the reference category among men for 15 year mortality from cardiovascular disease and coronary heart disease were 0.5 (95\% confidence interval 0.3 to 0.9 ) and $0.4(0.2$ to 0.8$)$, respectively (table II). For women associations were weaker and not significant, though they were based on small numbers of deaths. Fifteen year relative risks of ST depression $>0.25 \mathrm{~mm}$ for mortality from cardiovascular and coronary heart disease for men were $1.9(1.1$ to 3.0$)$ and $2.2(1.2$ to 3.9), respectively. Among women the corresponding risk ratios were $1.8(0.9$ to 3.6$)$ and $1.9(0.6$ to 6.3$)$. ST depression of $0.25 \mathrm{~mm}$ was not significantly associated with mortality. 
TABLE III-Case-cohort ${ }^{\star}$ relative risk of 28 year mortality from all causes, cardiovascular disease, and coronary heart disease by $S T$ segment level, with $S T$ isoelectric as reference category

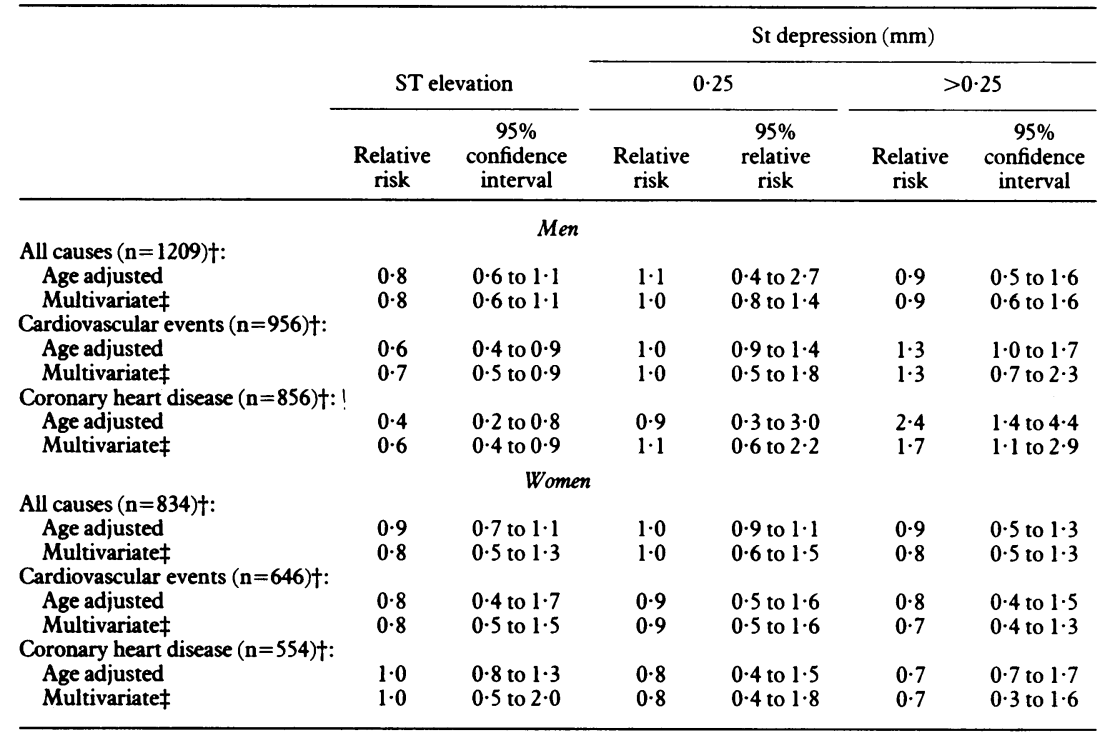

^Cases compared with random sample from cohort.

†Size of case-cohort population for given category of mortality.

$\ddagger$ Adjusted for age, total serum cholesterol concentration, diastolic blood pressure, smoking, and body mass index.

The pattern of the associations for 28 year mortality among men was similar, but, as expected, most associations were weaker. In the female population no clear predictive value was apparent over the full 28 year period (table III).

The strong inverse association for ST elevation among men persisted when men with evidence of manifest heart disease at baseline were excluded.

\section{Discussion}

In this 28 year follow up study slight ST elevation in standard lead I showed a strong inverse association with the risk of mortality from cardiovascular and coronary heart disease among men, particularly during the first 15 years of follow up. An increased risk was observed for ST depression $>0.25 \mathrm{~mm}$. Among women associations were weaker.

Several other investigators have reported an increased risk of mortality from coronary heart disease ${ }^{2-46}$ or of sudden death ${ }^{78}$ for more prominent ST depression in apparently healthy subjects, mostly men. However, they classified ST abnormalities according to the Minnesota code, ' which is not comparable with the detailed measurements in one lead performed in our study. We are not aware of any other study addressing ST elevation in a similar population.

Several potential methodological problems may have affected the validity of this study. The measurement and coding of ST segments, as well as the diagnosis and coding of causes of death, may have given rise to error. Moreover, a single measurement may be insufficient to characterise a person's average ST segment level. This may lead to non-differential misclassification, which increases with duration of follow up and results in dilution of associations; it also explains the weaker results after 28 years of follow up. A pharmacological effect on ST segment level at baseline is not likely because drugs that might affect the ST segment level were rarely taken in our sample.

Originally $54 \%$ of the candidate population volunteered to be examined, which is a low response rate and therefore raises doubts about the representativeness of the study population. The sampling procedure of the original population, however, did not intend to obtain a representative picture of all middle aged civil servants in the city of Amsterdam but rather to obtain a group of apparently healthy subjects. This may have affected the mortality observed but it probably did not influence the validity of the observed associations.

We used a case-cohort sampling design to limit the number of electrocardiograms to be coded. If the sample size is a small multiple of the size of the case series in this design then loss of precision is limited. ${ }^{11-13}$ A comparison of baseline characteristics between the cohort and the sample did not show substantial differences, so we consider the sample to be representative of the original cohort.

To check the impact of competing mortality we calculated crude and age adjusted incidence density ratios. These were similar for ST elevation and slightly higher for ST depression, compared with the reported cumulative incidence ratios.

In nearly all ST elevations the J point itself was not elevated or was considerably less elevated than the ST segment. This should be distinguished from J point elevation, often also reported as ST elevation, which indicates myocardial ischaemia or impaired left ventricular function and has a poor prognosis in patients with coronary disease. ${ }^{1718}$ Consequently, slight elevation, as measured in this study, in fact indicates a curved upwards slope of the ST segment, which has long been referred to as well shaped and been preferred to the isoelectric, stretched ST segment. Our results constitute the first empirical evidence supporting this preference. This protective effect is probably not the consequence of a lower prevalence of coronary heart disease at baseline in this category as the association persisted virtually unaltered when subjects with signs of manifest heart disease were excluded from the study population. The ST segment depressions, mostly slight, may indicate subclinical coronary disease or other conditions such as ventricular hypertrophy and electrolyte disturbances. ${ }^{19}$ Such undetected disease might explain the increased risk of mortality.

A different explanation of the findings could be that differences in ST segment levels in the study population are merely a consequence of autonomic function ${ }^{20}$ and the associations observed in this study are due to autonomic imbalance. Our results support previous findings indicating that ST segment depressions are more prevalent but less predictive in women. ${ }^{521}$

In conclusion, the kind of ST segment that by many physicians is intuitively appreciated for its good shape-a slight elevation at $80 \mathrm{~ms}$ after the $\mathrm{J}$ point indicates a low risk of mortality from cardiovascular and coronary heart disease in middle aged subjects after 15 years. ST segment depression on the contrary, indicates increased risk of mortality. Our results also show that usually neglected properties of the electrocardiogram of apparently healthy subjects may carry independent predictive information.

We thank Dr S Roelof $M$ van der Heide and Cornelia van der Heide-Wessel, the investigators from the original health study; Drs Simone van der Putten, Gerard Bles, Peter Nierop, and Jasper Schouten for coding the electrocardiograms; Dr Ale Agra for advice; and the Dutch Central Bureau of Genealogy and the Dutch Central Bureau of Statistics, for their help. The study was supported by grant 86.060 from the Netherlands Heart Foundation.

1 Prineas RJ, Crow RS, Blackburn H. The Minnesota code manual of electrocardiographic findings. Boston: John Wright, 1982.

2 Blackburn H, Raylor HL, Keys A. The electrocardiogram in prediction of five-year coronary heart disease incidence among men aged forty through fifty-nine. Circulation 1970;42 (suppl):154-61.

3 Rose G, Baxter PJ, Reid DD, McCartney P. Prevalence and prognosis of electrocardiographic findings in middle-aged men. $\mathrm{Br}$ Heart $\mathcal{f}$ 1978;40: 636-43.

4 Reunanen A, Pyörälä K, Punsar S, Aromaa A. Predictive value of ECG findings with respect to coronary heart disease mortality. Adv Cardiol 1978;21:310-2.

5 Liao Y, Liu K, Dyer A, Schoenberger JA, Shekelle RB, Colette P, Stamler J, et al. Major and minor electrocardiographic abnormalities and risk of death from coronary heart disease, cardiovascular diseases and all causes in men and women. $f$ Am Coll Cardiol 1988;12:1494-500. 
6 Ostrander $\mathrm{LD}$. The relation of "silent" $\mathrm{T}$ wave inversion to cardiovascular disease in an epidemiologic studv. Am 7 Cardiol 1970;25:325-8.

Cupples LA, Kannel WB. The elecirocardic . The Rabkin SW, Mathewson FAL, Tate RB. The electrocardiogram in apparently healthy men and the risk of sudden death. Br Heart $\mathcal{F}$ 1982;47:546-52.

9 Kagan A, Yano K, Reed DM, MacLean CJ. Predictors of sudden cardiac death among Hawaiian-Japanese men. Am $\mathcal{F}$ Epidemiol 1989;130:268-77.

10 Vandenbroucke JP, Matroos AW, Van der Heide-Wessel C, Van der Heide RM. Parental survival, an independent predictor of longevity in middle aged persons. Am J Epidemiol 1984;119:742-50

11 Miettinen OS. Theoretical epidemiology. Principles of occurrence research in medicine. Chichester: Wiley, 1985

12 Miettinen OS. Design options in epidemiologic research. An update. Scand $\mathcal{f}$ Work Environ Health 1982; suppl 1:7-14.

13 Miettinen OS. The "case-control" study: valid selection of subjects. f Chronic Dis 1985;38:543-8.

14 van der Heide RF. Resultaten van een geneeskundig onderzoek van 3091 gezonde mannen en vrouwen, 40-65 jaar oud [PhD thesis]. Amsterdam: University of Amsterdam, 1959. $143 \mathrm{pp}$.
15 Manual of the international statistical classification of diseases, injuries, and causes of death 9th ed Vol 1. Geneva: World Health Organisation, 1977.

6 (6)enland S. Adjustment of risk ratios in case-base studies (hybrid epidemiologic designs). Stat Med 1986;5:579-84

17 Fortuin NJ, Friesinger GC. Exercise-induced ST-segment elevation. Clinical, electrocardiographic and arteriographic studies in twelve patients. Am F Med 1979;49:459-64.

8 Bruce RA, Fisher LD, Pettinger M, Weiner DA, Chaitman BR. ST segmen elevation with exercise: a marker for poor ventricular function and poor prognosis. Coronary artery surgery study (CASS) confirmation of Seattle heart watch results. Circulation 1988;77:897-905.

19 Ellestad MH. Stress testing. Principles and practice. Philadelphia: F Davis, 1980:95-102.

20 Quyyumi AA, Wright C, Fox K. Ambulatory electrocardiographic ST segment changes in healthy volunteers. Br Heart f 1983;50:460-4.

21 Bruce RA, Irving JB. Exercise electrocardiography. In: Hurst W, ed. The heart, arteries and veins. 4th ed. New York: McGraw-Hill, 1978.

(Accepted 27 November 1991)

\title{
Intestinal effects of captopril
}

\author{
I Ralph Edwards, David M Coulter, \\ Donald Macintosh
}

\section{National Toxicology}

Group, Otago University

Medical School,

Dunedin, New Zealand

I Ralph Edwards, FRCP, medical assessor for medicines adverse reactions

David M Coulter, MB, assistant to the medical assessor Donald Macintosh, MB, senior lecturer in clinical pharmacology

Correspondence to: Professor I R Edwards, WHO Collaborating Centre for International Drug Monitoring, Box 26,

S-75103 Uppsala, Sweden.

BMF 1992;304:359-60
Captopril, an angiotensin converting enzyme inhibitor, should reduce all the effects caused by angiotensin II and III. Angiotensin affects the gut, ${ }^{1}$ and we report on six patients with adverse gastrointestinal effects that were associated with captopril and consistent with inhibition of some aspect of the agonist effect of angiotensin on intestinal function. We also review other similar cases reported to the World Health Organisation.

\section{Patients, methods, and results}

Captopril has been monitored by the New Zealand Medicines Adverse Reactions Committee through its intensive medicines monitoring programme since 1981 . The clinical events reported are analysed by at least two clinicians, and reports of new or serious events are followed up for an assessment of causality. The completeness of the cohorts from the monitoring of prescription data by pharmacists is about $85 \%$.

Out of a total cohort of 4124 patients, 169 were reported as having 220 events (mostly medicine related). Of these events, four $(1 \cdot 8 \%)$ were of gastrointestinal disturbance, at that time.

The table shows the clinical details of six patients reporting constipation and diarrhoea associated with taking captopril. The severity of the event was sufficient to prompt withdrawal of treatment in five of them, and causality with captopril was highly likely in all of them. In cases 1 and 2 the causal relation was strong because captopril was the only drug changed in the treatment and there was no confounding underlying illness. The temporal relations were feasible both for onset and for disappearance of symptoms. The striking feature was the severity of the condition. No treatment was given in either case, but the symptoms resolved soon after captopril was withdrawn. Diltiazem may have interacted with captopril in case 1 .
Three further cases of uncomplicated constipation were reported to the intensive medicines monitoring programme (three women aged $65-80$ taking $25-75 \mathrm{mg}$ captopril daily). In two no other medicines were used, and in none were symptoms severe enough to withdraw the drug. Forty three cases were recorded in the WHO's international database to the end of June 1991.

The four cases of diarrhoea in the table were probably due to captopril. Again, withdrawal of captopril resulted in rapid resolution of symptoms. In two patients captopril was the only drug taken. Another seven patients (three men, four women, age 46-87) taking $18 \cdot 75-150 \mathrm{mg}$ captopril daily had less severe diarrhoea (without vomiting in three patients). This did not result in withdrawal of captopril and causality was speculative. The WHO's database had 186 reported cases of diarrhoea to the end of June 1991.

\section{Discussion}

Angiotensin stimulates intestinal motility ${ }^{1}$ and its blockade may be expected to result in atony and constipation. Though the mechanism of this response is unclear, in rats prostaglandin inhibition failed to prevent the stimulating effects of angiotensin II. $^{2}$ In dogs Walus et al showed an angiotensin induced increase in intestinal activity in vivo with reduced blood flow, though oxygen consumption was unaltered. ${ }^{3}$ Inhibition of angiotensin converting enzyme should therefore reduce motility with an increase in blood flow and may be beneficial in intestinal (ileal) ischaemia as well as causing constipation.

Angiotensin II blocks the secretion of water and electrolytes in the ileum and colon in rats ${ }^{4}$; thus its inhibition would increase water and electrolyte concentrations in the intestine. Levens discussed a biphasic action of angiotensin II, low doses increasing sodium and water absorption from the intestine, high doses having the reverse effect. ${ }^{5}$ The low dose effect is secondary to local release of noradrenaline, but inhibition of absorption at high doses is thought to be due to prostaglandins. This biphasic dose-response makes prediction of the effects of angiotensin converting enzyme inhibitors difficult. Aldosterone, too, enhances colonic absorption.

Clinical details of six patients taking captopril who reported constipation and diarrhoea

\begin{tabular}{|c|c|c|c|c|c|c|c|c|}
\hline $\begin{array}{l}\text { Case } \\
\text { No }\end{array}$ & Sex & $\begin{array}{c}\text { Age } \\
\text { (years) }\end{array}$ & $\begin{array}{l}\text { Daily dose } \\
\quad(\mathrm{mg})\end{array}$ & $\begin{array}{l}\text { Duration of treatment } \\
\text { before onset (days) }\end{array}$ & Other drugs & Indication & Comment & $\begin{array}{l}\text { Time till resolution } \\
\text { after stopping captopril }\end{array}$ \\
\hline \multicolumn{9}{|c|}{ Constipation } \\
\hline 1 & $\mathbf{F}$ & 61 & 25 & 21 & Diltiazem & Congestive heart failure & Atonic bowel; megacolon & 3 days \\
\hline 2 & $M$ & 44 & 75 & 7 & None & Hypertension & Complete bowel atony & 24 hours \\
\hline \multicolumn{9}{|c|}{ Diarrhoea } \\
\hline 3 & $M$ & 62 & 75 & 17 & None & Hypertension & With vomiting & 24 hours \\
\hline 4 & $\mathrm{~F}$ & 43 & $12 \cdot 5$ & 28 & None & Hypertension & With vomiting & Treatment not stopped \\
\hline 5 & $\mathrm{~F}$ & 74 & 25 & & \multirow{3}{*}{$\begin{array}{l}\text { Frusemide, sulindac, } \\
\text { ethinyloestradiol, alginic acid } \\
\text { (Gaviscon) } \\
\text { Cimetidine, prazosin }\end{array}$} & & & \\
\hline & & & & & & Congestive heart failure & With vomiting & 2 days \\
\hline 6 & $M$ & 61 & 25 & First dose & & Hypertension & Abdominal distension & 24 hours \\
\hline
\end{tabular}

